\title{
Commissioning and Initial Operation of the W7-X Neutral Beam Injection Heating System
}

\author{
P. McNeely ${ }^{a^{*}}$, S. Äkäslompolo ${ }^{a}$, W. Auerweck ${ }^{c}$, Y. Drider $^{\mathrm{a}}$, O.P. Ford ${ }^{\mathrm{a}}$, D.A. Hartmann ${ }^{\mathrm{a}}$, B. \\ Heinemann $^{\mathrm{b}}$, S. Heinrich ${ }^{\mathrm{a}}$, C. Hopf ${ }^{\mathrm{b}}$, R. Kairys ${ }^{\mathrm{a}}$, S. Obermayer ${ }^{\mathrm{b}}$, R. Riedl ${ }^{\mathrm{b}}$, P. Rong ${ }^{\mathrm{a}}$, N. Rust ${ }^{\mathrm{a}}$, R. \\ Schroeder ${ }^{\text {a }}$ R.C. Wolf ${ }^{\mathrm{a}}$, and the W7-X Team ${ }^{[1]}$ \\ ${ }^{a}$ Max-Planck-Institut für Plasmaphysik-Teilinstitut Greifswald, Wendelsteinstrasse 1, 17391 Greifswald, Germany \\ ${ }^{b}$ Max-Planck-Institut für Plasmaphysik, Boltzmannstrasse 2, 85748 Garching, Germany \\ cSiemens AG, München, Germany
}

\begin{abstract}
The first, of two planned, neutral beam injectors for the stellarator Wendelstein 7-X (W7-X) was commissioned for and participated in the experimental campaign (OP1.2b) from July to October 2018. The injector was equipped with two RF driven ion sources from which $90 \mathrm{~A}$ of positive hydrogen ions were extracted at $55 \mathrm{kV}$. After neutralization, the two sources provided $>3 \mathrm{MW}$ of neutral beam heating power to the stellarator plasma. During the experimental campaign $>300$ shots were successfully performed for plasma heating or to allow for measurement of the ion temperature profile by charge exchange recombination spectroscopy (CXRS). The initial operation of the NBI system on W7-X was very successful, demonstrating both increased central plasma density and stored plasma energy, or allowing for the collection of the time resolved ion temperature over the bulk of the plasma. This paper presents, briefly, source conditioning and performance, focusing on some of the initial problems encountered. Described in more detail is the most significant challenge overcome during commissioning: failure to couple RF power into one of the sources.

Keywords: NBI, Ion Source, Plasma Heating
\end{abstract}

\section{Introduction}

The Stellarator W7-X completed its initial operation phase with an uncooled divertor in 2018 [2] and is currently undergoing installation of the water cooled long pulse capable divertor in preparation for the next experimental campaign. In the last part of the initial operational phase (designated OP1.2b, which was between July and October of 2018) the first injector (NI21) of the neutral beam heating (NBI) system was successfully commissioned [3, 4].

The NBI injector for W7-X (see Figure 1) is a copy of the Asdex Upgrade (AUG) [5] $2^{\text {nd }}$ injector [6-10] in terms of most of the injector components (vacuum chamber, ion sources, grids, neutralizers, ion dumps and deflection magnet). However, the calorimeter [11] and parts of the ion dumps are, based on operational experience at AUG, modified to improve performance and ease of repair. Moreover, the support systems [12] for the NBI system (cooling water, RF generators, control system, safety system, high voltage power supplies for the ion sources, etc.) plus the duct, its protection [13] and the beam dump protecting the inner wall of $\mathrm{W} 7-\mathrm{X}$ are all new.

The injector NI21 was equipped with 2 of 4 possible ion sources. The sources are operated in hydrogen to provide $90 \mathrm{~A}$ of extracted ion current $\left(\mathrm{H}^{+}, \mathrm{H}_{2}{ }^{+}, \mathrm{H}_{3}{ }^{+}\right)$at an acceleration voltage of $55 \mathrm{keV}$. The grids used were conditioned on the AUG $1^{\text {st }}$ Injector prior to transport to W7-X and installation in the PINI (Plug In Neutral Injector) for the source. Each source is driven by a TRANSRADIO SCIAMP $150 \mathrm{~kW} 1 \mathrm{MHz}$ solid state amplifier rather than the Himmelwerk Generator used on AUG. The initial pair of ion sources selected for operation (Source 7 and Source 8) were chosen as one (source 8) is a tangential source with good duct transmission and the other one (source 7) a more radial source [14] that makes the mechanical assembly as simple as possible.

\section{Source Commissioning}

The sources used at W7-X are the standard AUG Type 2 ion sources $[15,16]$. The bodies of the sources were newly produced for W7-X NBI, while the acel-decel triple grids were originally reserve sets for AUG. The grids have 774 apertures with $8 \mathrm{~mm}$ diameter arranged in two half-grids, with the grid halves inclined by an angle of $0.873^{\circ}$ to the horizontal giving a focal distance of $8.5 \mathrm{~m}$. The middle grid (Decel-grid) is biased negative compared to the last grounded grid via a bias resistor to provide suppression of the back streaming electrons from the neutralizer. Before source operation with beam, the grids were conditioned under gas to $60 \mathrm{kV}$.

As there was a requirement to perform safety testing covering high voltage aspects of NBI operation it was required to generate high voltage breakdowns to confirm that they would have no negative consequences on $\mathrm{W} 7-\mathrm{X}$ or personnel in the torus hall. Source 7 was, at this time point, not in operation (see section 4) and was used for this task. It was then observed that although it was possible to bring Source 8 into operation with a limited number of beam extraction shots, source 7 took significantly more time to achieve the same operation point. It seems a reasonable assumption that forcing the grids of source 7 to generate breakdowns for test purposes is the cause of this disparity in conditioning shot numbers. 
Further, based on AUG experience, it was expected that the RF power required for nominal operation would be $\sim 1.3 \mathrm{~kW}$ RF power per Ampere extracted beam. Thus the RF generators, when purchased, were limited to 125 $\mathrm{kW}$ maximum output power. Early conditioning on source 8 revealed that the RF power requirements were higher than expected $(\sim 1.5 \mathrm{~kW}$ RF power per Ampere extracted beam). This required the power limitation to be removed; allowing the generators full $150 \mathrm{~kW}$ capability to be utilized. The higher required RF power is, in part, due to operation of the source with a $20 \%$ lower gas flow.

A consequence of the lack of a fast high voltage switching control between the power supply and the ion source, as is realized on AUG via a high power tetrode, was also found during initial operation. The issue was that the return path of the ion source current was connected across the potential free outputs of the power supply. The high voltage applied over the grid stack was therefore not properly taking into account the negative (electron suppression) voltage applied to the middle or
Decel grid. As the electron suppression potential is provided by a voltage divider arrangement (Bias Resister) to provide the proper acceleration voltage required the operator to manually increase the requested high voltage by the amount of the suppression voltage based on the expected value from the resistor. This solution worked successfully and there proved to be no difficulty operating the grids at the higher potential.

On 31.05.18 Source 8 achieved a plasma generated by $45 \mathrm{~kW}$ for $5 \mathrm{~s}$. Source 8 was available for injection into $\mathrm{W} 7-\mathrm{X}$ at reduced parameters $(46 \mathrm{kV}, 67 \mathrm{~A})$, due to the limitations of available RF power, on 5.07.18. Source 7's first successful plasma generation and beam extraction was on 06.07.18. Source 7 achieved $46 \mathrm{kV}$ on 25.07.18. First NBI injection into a W7-X plasma (both sources 25 $\mathrm{ms}$ ) was on 08.08.18. It was possible to operate Source 8 at full parameters on 30.08.18; Source 7 followed on 11.09.18.

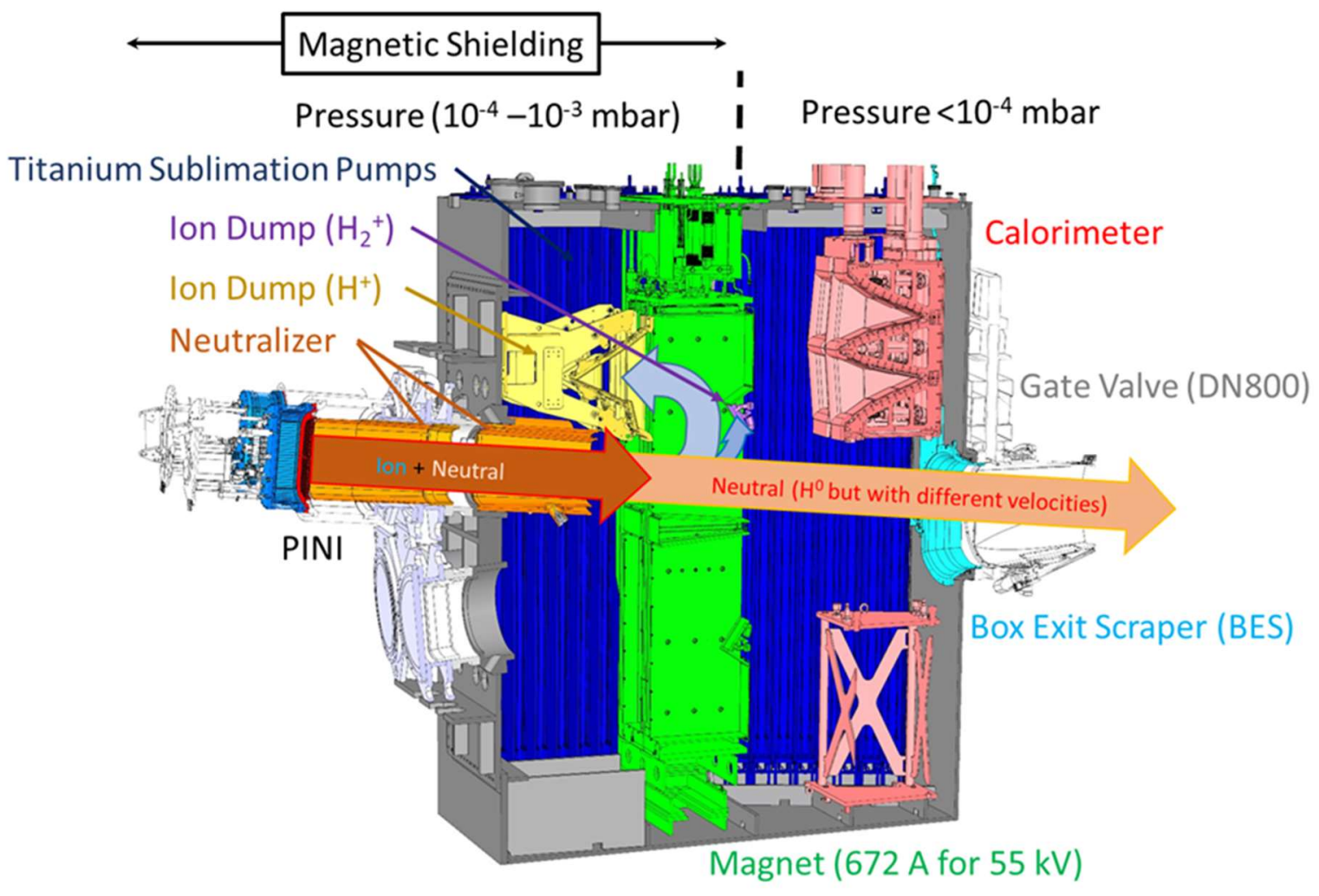

Fig. 1. Figure showing the main components of the neutral beam injector NI21.

\section{Performance}

Both sources performed very well over the experimental campaign once the limitations of and worka-rounds to them were established for the main W7-X NBI segment control system. It was possible for the sources to be operated in continuous heating pulses (up to $5 \mathrm{~s}$ ), short beam blips ( $<30 \mathrm{~ms}$ pulses) with low duty cycle for determination of the ion temperature via charge exchange recombination spectroscopy, interwoven beam pulses from both sources or with power modulation via duty cycle variation. Shots could be performed with the Electron Cyclotron Heating (ECRH) system or sustained by NBI alone with the ECRH system necessary for plasma startup discontinued.

Based on the initial results of a study of failed attempts to inject into W7-X, 38 of 302 injection attempts failed, excluding failures where $\mathrm{W} 7-\mathrm{X}$ terminated the plasma early, giving an injector reliability of $>85 \%$. The vast 
bulk of these 38 failures appear to be due to a failure in the programing of the segment control system by the experiment leader. As tools were developed over the campaign to reduce the chance of these failures, the reliability of NBI injection increased over the course of OP1.2b.

For source 8, the performance of the source in terms of extracted current at a given extraction voltage is shown in Figure 2. It can be seen that the source was largely operated in an over perveant mode, this was due to the issues with the power supply voltage combined with a further problem which became apparent during commissioning, namely that the signals from the high voltage power supply for the high voltage value (either sent as a direct analogue signal or sent as a digital signal over the network) were in error. For the analogue signal a new calibration for the high voltage value was derived based on the requested high voltage value and the signal returned from the power supply. The digital signal was both time shifted and suffered from significant signal distortion rendering it useless.

Comparing the high voltage applied to the value of beam energy derived from neutralizer spectroscopy indicates the energy of the beam measured in the neutralizer is higher, in all cases so far checked, than the calculated extraction voltage based on the requested high voltage and the measured bias voltage. The reason for this discrepancy is still under investigation, but so far no explanation has been found.

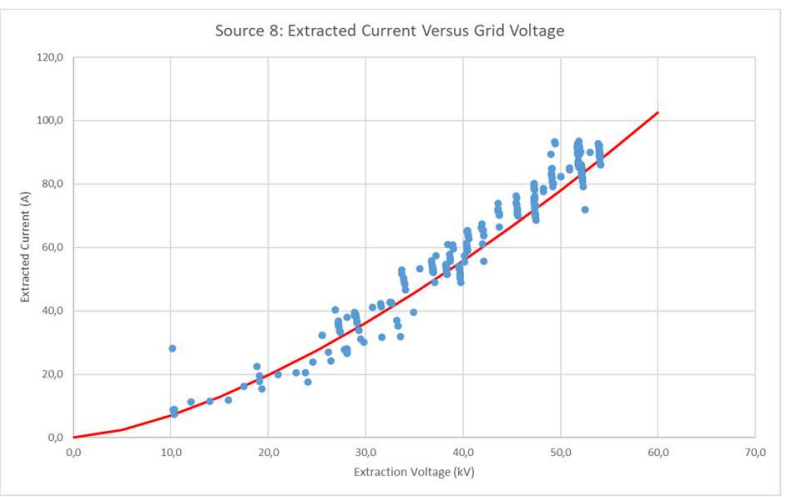

Fig. 2. Extracted current as a function of Acceleration Voltage for source 8 . The solid line is the theoretical curve for a perveance of $8.69 \times 10^{-6} \mathrm{~A} / \mathrm{V}^{3 / 2}$ at $55 \mathrm{keV}$.

The current extracted from the source was measured to +-0.7 A based on measurements of the accuracy of the installed LEM inductive current measurement system at the injector. The high voltage applied to the source can be in error by up to $2 \mathrm{kV}$ from what requested from the power supply as determined by comparing the spectroscopically measured beam energy to the values supplied by the power supply. The lack of accurate knowledge of the value of the high voltage applied to the grids has consequences for the operation of the grids, as to prevent damage they need to be operated near to the theoretical perveance optimum, shown in figure 2 as a solid line.

\section{Challenge: Matching Source 7}

The ion sources used on the W7-X injector are driven by inductively coupling the power from the RF generators into the plasma via a Faraday shield screened antenna. This makes achieving plasma ignition in the ion source the initial goal of the commissioning process. In Figure 3 is shown the matching network for coupling the power into the plasma. The process is performed in the straightforward way of estimating the required value of $\mathrm{C} 1$ and then sweeping $\mathrm{C} 2$ or the generator frequency until the plasma ignites reliably determined by observing both the reflected RF power and the observed plasma light on a diode. Experiments were performed in Garching, on a different ion source, to confirm the initial estimate of $\mathrm{C} 1$ and this value of $1.43 \mathrm{nF}$ was used as the starting conditions for ignition at $\mathrm{W} 7-\mathrm{X}$.

The initial attempts to ignite either source were unsuccessful. During these attempts monitoring of the RF signals with a fast oscilloscope was showing strange wave form present on the RF start/stop signal. Further investigation showed that the waveform was coming from an improperly chosen couple relay on the input output board which required a higher minimum current then the board could deliver. Exchange for one which matched the current capabilities of the board solved this problem. Also observed were abrupt termination of the RF with the generator control stating that the reflected power was excessive, unfortunately this was not observed in the data which only showed the RF power terminating.

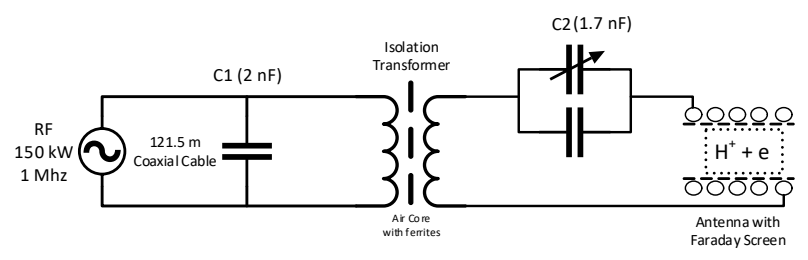

Fig. 3. The matching network of the RF sources. The inability to ignite source 7 was due to reduced voltage holding capability of the fixed part of the C2 vacuum capacitor.

The questions of both the failure of either source to match and the abrupt termination of the generator remained. The possibilities for both were: the value of C1 was wrong, there was something wrong with the matching system, the generator was the cause, the control system had a bug in it, or operator error. The generator was tested on a dummy load and worked as expected, excluding the generator. The matching network was visually inspected and all deficiencies were corrected to ensure all connections were made with well clamped, large surface area contacts. The new control system was excluded as a source of error after it became possible to run a badly matched plasma, at low power, to the maximum pulse length planned. This left the value of $\mathrm{C} 1$ as the sole remaining possibility for the lack of matching.

It was determined by W. Kraus (IPP Garching) that an increase of $\mathrm{C} 1$ by $50 \%$ would be a likely matching point. The value of $\mathrm{C} 1$ was increased to $1.8 \mathrm{nF}$ as a test and 
within a few ignition attempts it was very clear that the matching was improved significantly but was still not sufficient to ignite the source and that $\mathrm{C} 1$ needed to be again changed. The speed at which it was possible to conclude a change was required indicated that a substantial part of the initial delay was due to human factors plus the new confidence of the operator in the proper working of the control system. These human factors are ones that any group working with the remote assistance of another group are likely to face. Being responsible for determining matching on a RF system teaches what is required for the matching in a way that observing someone else doing the task cannot. Second guessing is reduced with experience and positive results. Finally, the confidence must be present that all the system components are operating correctly. This point, especially when dealing with a completely new system, as in the case of W7-X, is where the primary challenge to the operator lies.

The value of $\mathrm{C} 1$ was raised to $2 \mathrm{nF}$ and it proved possible to match source 8 , but source 7 stubbornly failed to ignite. The generator for source 7 failing with "bad matching" or demonstrating the same poor matching that was present with an incorrect setting of $\mathrm{C} 1$. Investigation of the matching network could determine no difference between the two sources. One worked while the other did not. Utilizing the generator and transmission line, up to the isolation transformer, for source 8 on source 7 did not enable ignition.

The investigation into source 7's problem ended when it was observed that irrespective of the matching conditions, if the forward power was above a certain value the generator would fail with "bad matching" while below that power level it would be possible to run the discharge in a poorly matched state. In general, for this power level the RF current is approximately constant impendent of the power while the RF voltage varies with power. Therefore it was concluded that below a certain voltage the system was functioning (albeit poorly) while above that voltage something was arcing over triggering the generator failure.

The voltage holding capacity of all elements of the matching circuit were tested and a failure of the fixed component of the variable vacuum capacitor $\mathrm{C} 2$ was found. This component had been purchased some 10 years prior to installation on source 7 . The potential for the cause of the problem to be voltage holding had been suggested earlier in the investigation but due to the component being "new" it was deemed to be very unlikely to be the culprit. After replacement matching and testing were quickly completed and about 2 hours after achieving $40 \mathrm{~kW}$ plasmas in the source beam had been extracted at $18 \mathrm{kV}$.

During the process of upgrading the generators to 150 $\mathrm{kW}$ discussions with the manufacturer revealed that the extremely fast turn off of the generators was a design feature intended to protect the antenna. It also turned out that a common question from their customers was: why had the generator turned off without any indication of a problem in the signals visible to the operators?

\section{Results with W7-X}

The first and most important result of use of NBI on W7-X was that it proved possible to sustain the plasma with NBI alone for up to $5 \mathrm{~s}$, regardless of the limited power available $(\sim 3.4 \mathrm{MW})$ compared to the size of the stellarator. The operation of the NBI also was shown to not disturb the operation of the machine (in terms of gas load, RF generators, $\mathrm{HV}$, etc.).

The use of NBI allowed operation of W7-X to central electron densities of almost $2 \times 10^{20} \mathrm{~m}^{-3}$, a value beyond the ECRH systems X2 cutoff $\left(1.2 \times 10^{20} \mathrm{~m}^{-3}\right)$. The NBI also allows for the possibility of $\mathrm{O}-\mathrm{X}-\mathrm{B}$ Heating (mode conversion from $\mathrm{O}$ to $\mathrm{X}$ to Bernstein waves) by the ECRH system where the NBI can be used to bridge the density gap.

Additionally, the NBI served as a critical diagnostic tool. It was a neutral source for charge exchange spectroscopy, which provided high precision ion temperature measurements $[17,18]$, the first absolute impurity density profiles at W7-X [19] and first local radial electric field measurements in the plasma core [20].

\section{Conclusion}

The NBI system was successfully commissioned on $\mathrm{W} 7-\mathrm{X}$ in OP1.2b. Injection at the planned source parameters was achieved $(55 \mathrm{keV}, 90 \mathrm{~A})$ for up to 5 seconds dependent on the limits established based on calculated fast ion power deposition for each magnetic configuration of W7-X. In addition to heating the plasma, either alone or in conjunction with the ECRH system the NBI enabled routine measurement of ion temperature via short beam blips for the CXRS system. The use of NBI also produced a peaked plasma profile and the highest central density recorded so far in W7-X $\left(2 \times 10^{20} \mathrm{~m}^{-3}\right)$.

This paper also detailed the difficulties encountered in getting source 7 into operation. It is an important lesson for other machines in the future as it illustrates a number of points. Primarily, the need to train operators on as nearly identical a test bed as possible. Efficient fault finding requires a familiarity with the specifics of the system, as can be demonstrated by the as designed fast turn off of the generators in response to a poor match, not one visible to the operator. Knowing this detail earlier would have saved a considerable amount of time and effort in investigating a system that was, in fact, working properly and as intended.

The human aspect of commissioning is also critical, for example, unless the operators have confidence in the proper functioning of the control system it is easy to assign every failure to it. Furthermore, commissioning is a process requiring good group dynamics and teamwork where people feel free to contribute their ideas. The path that lead to determining the cause of the matching issue was acceptance of a suggestion to perform a test that was not sensible to perform: increasing the applied power even though the matching was obviously wrong. Another point 
that should be kept in mind is that as virtually all systems were new, when it was necessary to failure search, the time it took was increased significantly. Due mostly to the fact it was not immediately possible to exclude elements from the list of possible causes of the failure.

The commissioning of the second injector NI20 with two sources will occur late in 2020 or early in 2021. For the next campaign of W7-X there will be four ion sources available (total of $6 \mathrm{MW}$ heating power) and the possibility of balanced injection. It is also planned to increase the length of time the injector can provide "beam blips" for diagnostic purposes to as long as the vacuum in the box can be held to acceptable values (15-30s).

\section{Acknowledgments}

This work has been carried out within the framework of the EUROfusion Consortium and has received funding from the Euratom research and training program 20142018 and 2019-2020 under grant agreement number 633053. The views and opinions expressed herein do not necessarily reflect those of the European Commission.

\section{References}

[1] Klinger T et al 2019 Nuclear Fusion 59112004 doi: 10.1088/1741-4326/ab03a7

[2] Wolf RC et al 2019 Physics of Plasmas 26, 082504; https://doi.org/10.1063/1.5098761

[3] Hartmann DA et al 2019 46 ${ }^{\text {th }}$ European Physical Society Proceedings Milan Italy P1.1076

[4] Rust N et al 2019 46 ${ }^{\text {th }}$ European Physical Society Proceedings Milan Italy P5.1057

[5] Zohm H et al 2009 Nucl. Fusion 49104009

[6] Stäbler A et al 1989 Proceedings of the 15th Symposium on Fusion Technology ed AM Van Ingen et al (Amsterdam: Elsevier Science) 620

[7] Stäbler A et al 1995 Proceedings of the 18th Symposium on Fusion Technology ed K Hershback et al (Amsterdam: Elsevier Science) 593

[8] Vollmer O et al 1993 Proceedings of the 17th Symposium on Fusion Technology ed C Ferro et al (Amsterdam: Elsevier Science) 1106

[9] Vollmer O et al 1998 Proceedings of the 20th Symposium on Fusion Technology ed M Gasperotto and P Komarek (Amsterdam: Elsevier Science) 449

[10] Heinemann B et al 1993 Proceedings of the 17th Symposium on Fusion Technology ed C Ferro et al (Amsterdam: Elsevier Science) 524

[11] Holtum D et al 2011 Fusion Eng. Des. 86905

[12] McNeely P et al 2013 Fusion Eng. Des. 881034

[13] Nocentini R et al 2015 Fusion Eng. Des. 100453 doi:10.1016/j.fusengdes.2015.07.016.

[14] Rust N et al 2011 Fusion Eng.Des. 86728

[15] Speth E et al 1999 Fusion Eng. Des. 46383

[16] Kraus W et al 1998 Rev. Sci. Instr. 69956

[17]Ford OP et al 2019 46 $6^{\text {th }}$ European Physical Society Proceedings Milan Italy P2.1051

[18] Bozhenkov SA et al 2019 to be submitted to Nuclear Fusion
[19] Vanó L et al 2019 46 $6^{\text {th }}$ European Physical Society Proceedings Milan Italy P5.1065

[20] Alonso A et al $201946^{\text {th }}$ European Physical Society Proceedings Milan Italy I3.102 